\title{
Evaluation of Coded Excitations for Autonomous Airborne Ultrasonic Inspection
}

\author{
Dayi Zhang, Robert Watson, Gordon Dobie, Charles MacLeod, David Lines, \\ Walter Galbraith, Carmelo Mineo, Gareth Pierce \\ Centre for Ultrasonic Engineering \\ University of Strathclyde \\ Glasgow, United Kingdom \\ dayi.zhang@strath.ac.uk
}

\begin{abstract}
Unmanned Aerial Vehicles (UAVs) are receiving increasing attention for use in Non-Destructive Testing due to their ability to access areas where manual inspection is not practical. Contact-based UAV ultrasonic inspections grant the opportunity to remotely monitor the structural health of an industrial asset with enhanced internal integrity information. Ultrasonic inspection is a Non-Destructive Testing (NDT) method conventionally used in corrosion mapping. Surface contacting ultrasonic transducers provide enhanced structural integrity information. However, due to near-surface aerodynamic effects, angular sensitivity of the ultrasound probe and alignment error during autonomous inspections, ultrasonic thickness measurements with low Signal-to-Noise Ratio (SNR) are common. Coded excitation consists of a series of binary bits, whereby the polarity of the voltage is varied following a set excitation sequence to produce desirable signal properties. Here, coded excitation is utilised to increase SNR and thereby reduce measurement uncertainty originating from non-ideal transducer alignment with asset surfaces during inspections. This paper evaluates the performance of two binary code sequences (8-bit Golay, 13-bit Barker) for use in autonomous airborne inspections.

Index Terms-Coded Excitation, Ultrasonic Inspection, UAV
\end{abstract}

\section{INTRODUCTION}

A UAV (Unmanned Aerial Vehicle) is a pilotless aerial robot system, generally comprised of a flight controller, navigation and communication system and a functional payload. Their mobility and size grant UAVs the ability to effectively undertake many dangerous tasks, such as NDT (Non-Destructive Testing) inspections. The current state of the art in the field of UAV based NDT focuses on photogrammetric [1], thermographic [2], and ultrasonic inspections [3]. Previous research has demonstrated an autonomous, multi-rotor UAV deployed, contact-based, ultrasonic measurement system for the contact inspection of non-magnetic facilities. Inspections of an unpainted, vertically mounted, aluminium sample were conducted by the UAV in an indoor laboratory environment [4].

However, as is discussed in [4], a number of factors were observed to influence the system performance when applied to the context of UAV-based inspection. Transducer alignment

This research is funded by the ESPRC Autonomous Inspection in Manufacturing \& Remanufacturing (AIMaReM) project (EP/N018427/1) and Pressure Profile Systems, Inc. (PPS). errors and UAV instabilities cause poor acoustic coupling, exhibited as high attenuation of the returned ultrasonic signals.

Increasing the excitation voltage applied to the piezoelectric element is an example of a strategy used to lessen this challenge. Doing so propagates more energy into the test sample and in turn ensures that the system receives stronger reflected waves. In this context, however, a high voltage system will increase the complexity of the electronics design. Averaging across a set of replicate signals is another method by which SNR may be improved. However, such a method requires the probe to be near stationary during the entire signal acquisition time, a challenge when subject to aerodynamic disturbances.

Therefore, a more elegant solution is sought in the use of coded excitation to increase the SNR within the UAV thickness measurement system. Coded excitation has previously been demonstrated within laser ultrasound applications [5] and ultrasonic inspections [6]. The excitation sequence consists of a series of bits coded as \pm 1 . Multiple pulses are transmitted in series whereby the polarity of the excitation voltage used for each pulse is varied according to bits of the excitation sequence. Coded excitation improves the received SNR without increasing the amplitude of the excitation voltage applied to the piezoelectric material and so is more suitable for the UAV system. Compared with signal averaging, it shortens the signal acquisition time necessary to achieve the same level of SNR improvement [5].

The Golay and Barker encodings are two types of binary sequence commonly applied to ultrasonic excitation [5]. Golay code excitation contains a pair of sequences, coded with two different polarity patterns. The two complementary signals received after the transmission of the coded sequences contain different wave patterns so that the sidelobes present in the resultant signal can be suppressed by summation. However, an approach using the Golay excitation scheme requires two measurements to be conducted in the same position and with similar coupling, and so may be impractical given the challenges associated with the UAV control system and environmental disturbance rejection. Barker coded excitation is more easily implemented in this context as it executes only one coded sequence, eliminating issues associated with small systemic changes during measurement at the expense of the 
sidelobe cancellation property present in Golay codes.

This paper evaluates the performance of the Golay and Barker code sequences for use in autonomous airborne inspections.

\section{Methodology}

\section{A. Golay code}

The Golay code is a sequence composed of a pair of complementary excitations, each with a length of $L=2^{n}$ bits. These signals have properties such that the sum of the autocorrelation functions of these two excitations is zero except at the point of zero lag, where the amplitude is maximised $(2 L)$ [7]. The complementary sidelobes near the echoes appearing in each transmitted signal's autocorrelation function are suppressed by superposition in the final result.

However, two sequence reflections might be executed and acquired with differing transducer alignments and positions during the UAV deployed ultrasonic measurements due to the near-surface aerodynamic challenges and flight controller limitations. This may result in discrepancies between two transfer functions and introduce errors to the summation of the complementary signals. Fig. 1 presents the 8-bit Golay coded sequence where $\mathrm{f}$ is the central excitation frequency $(5$ $\mathrm{MHz}$.

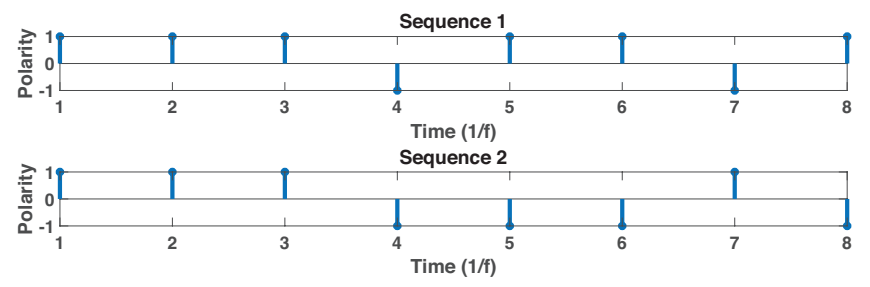

Fig. 1. A pair of 8-bit Golay sequences $(L=8)$ as employed herein

\section{B. Barker Code}

By contrast, the Barker coded excitation scheme requires only a single transmission sequence [8]. Similar to the Golay code, autocorrelation is used to recover the Barker coded waveform. Its autocorrelation function presents with amplitude $L$ at zero lag, with amplitude smaller than 1 elsewhere.

The single excitation of the Barker sequence mitigates issues associated with systemic changes during acquisition but, in doing so, the sidelobes in the autocorrelation function cannot be cancelled. Therefore, the SNR increase conferred by this approach is diminished, in turn offering a lesser enhancement of the transducer's resolution. In spite of this, using only one excitation provides the Barker code with sufficient robustness to function in the presence of minor systemic changes during signal acquisition and so allows its benefits to be applied to the current UAV deployed ultrasonic technologies. Fig. 2 presents the 13-bit Baker coded sequence used here.

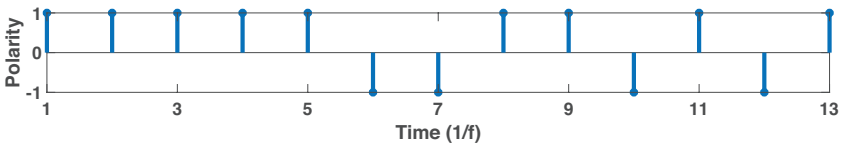

Fig. 2. A 13-bit Barker sequence $(L=13)$ as employed herein

\section{Ultrasonic Thickness Measurement}

Ultrasonic thickness measurement is attained utilizing a spring-loaded, $5 \mathrm{MHz}, 10 \mathrm{~mm}$-diameter, dual-crystal transducer manufactured by GB Inspection [9] and driven by a custom designed, low from-factor transceiver. A thin layer of couplant gel is applied between the sample and probe surfaces to eliminate any air gap prior to executing the motion path.

A $500 \times 500 \times 15 \mathrm{~mm}$ aluminium plate, including variations in thickness and simulated defects, was fabricated and vertically mounted in the measurement volume to mimic an industrial inspection scenario. (a)

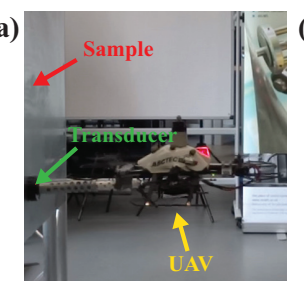

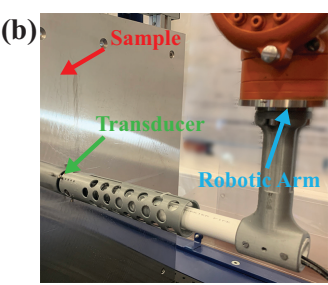

Fig. 3. Photos of complete setup when the transducer was deployed by (a) UAV (b) KUKA manipulator

The transducer was mounted on the end of a KUKA robotic manipulator arm, as shown in Fig. 3(b). Compared with the levels of vibration when mounted on the UAV, as per Fig. $3(\mathrm{a})$, the robotic manipulator can deploy the transducer with a far finer degree of position control, granting experimental repeatability. This method also allows for the isolation of larger scale motion features from vibration effects of the UAV propulsion for a more informative characterization procedure. The manipulator arm was controlled by the Interfacing Toolbox for Robotic Arms (ITRA) [10] to precisely deploy the transducer, mimicking a UAV flight trajectory recorded during a previous UAV contact inspection, as shown in Fig. 4. In the original flight trial, the transducer was intended to take a single point measurement. However, due to the near-surface aerodynamic challenges and flight controller limitations, the transducer pose was observed to vary while in contact with the sample surface. In the recorded flight dataset, the maximum translational and angular velocities of the UAV were 128.21 $\mathrm{mm} / \mathrm{s}$ and $14.46 \% \mathrm{~s}$ respectively.

In terms of signal generation and acquisition, the transmitting element inside the probe is activated by the ultrasonic transceiver, which is capable of both ultrasonic signal generation and acquisition. It executes $\pm 12 \mathrm{~V}$ bipolar rectangular pulse generation, vibrating the piezoelectric crystal element, and thus generating ultrasound pulses. Custom software running on the UAV onboard computer drives the transducer with a $60 \mathrm{~Hz}$ pulse repetition frequency, as dictated by the execution 

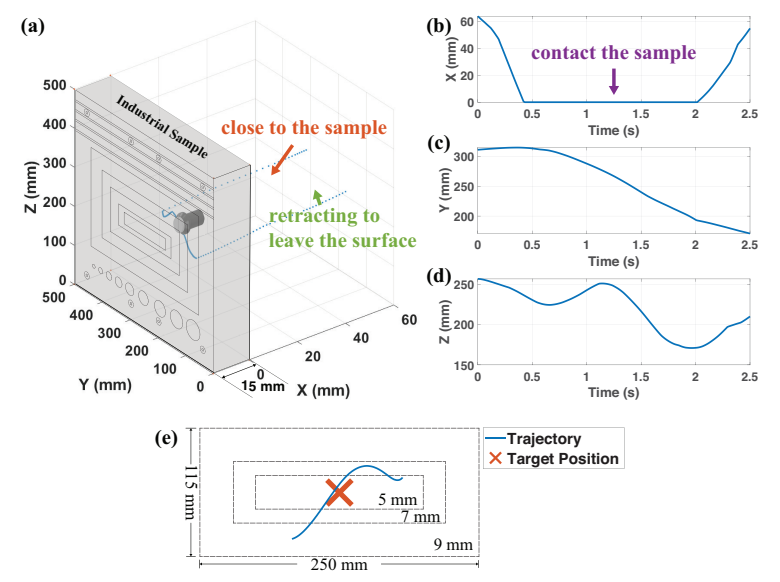

Fig. 4. (a)(b)(c)(d) The UAV flight trajectory recorded during a previous UAV contact inspection. (e) Detailed view of probe trajectory while in contact with the sample surface.

time for the real-time signal processing used to decode the Golay or Barker sequences and extract the thickness measurement from each resulting A-scan signal. As the Golay code is sensitive to transducer alignment and position, the two component sequences of Fig. 1 are transmitted in series within a period of $2 \mathrm{~ms}$ to minimise the error from transducer motion. To this end, the signal processing necessary to decode the received signals is conducted only after each pair have been digitised. Further processing then provides a quantitative Ascan thickness measurement.

\section{SNR Improvement}

To quantify the improvements offered by the Barker and Golay code sequences and the effects of the sidelobes produced by the Barker sequence, the transducer was held in stationary contact with the sample under ideal alignment and stimulated using the 13-bit Barker and 8-bit Golay coded excitations. For comparison, a standard single pulse excitation was also applied to the transducer using the same hardware. All returning A-scan signals (as shown in Fig. 5) were digitised by the ultrasonic transceiver, before being recovered from their encoded signal.

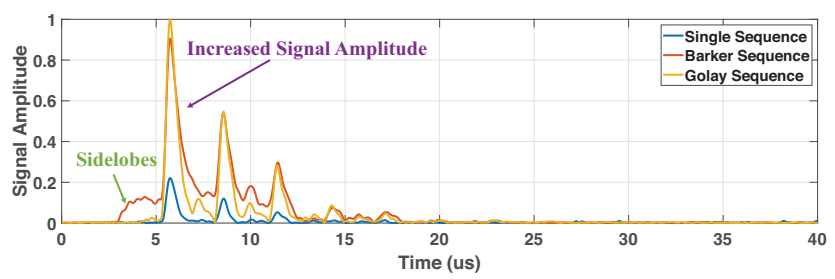

Fig. 5. A-scan signals of single pulse excitation, 13-bit Barker coded excitation, and 8-bit Golay coded excitation. The transciever amplifier gain was held at $13 \mathrm{~dB}$ throughout. Sample nominal thickness was $9 \mathrm{~mm}$.

As shown in Fig. 5, the signal amplitude was substantially increased by utilising the coded excitation. The SNR is calculated by dividing the peak amplitude of the reflected signal by the noise amplitude and expressing the result in decibels. The noise is taken as the maximum amplitude of the signal within the regions where the reflected echo is not observed, ignoring the initial pulse transmission. The SNR was increased by $10.55 \mathrm{~dB}$, from $35.37 \mathrm{~dB}$ in the single excitation to $45.92 \mathrm{~dB}$ under a 13-bit Barker coded excitation. Similarly, the SNR was increased to $48.82 \mathrm{~dB}$ in 8-bit Golay coded excitation. The values agree with those expected from established theory. Compared with the Golay excitation, the Barker sequence presented a similar signal amplitude but sidelobes were observed near the back-wall echoes which present a challenge to some signal processing algorithms used to extract a numerical thickness value.

\section{RESUlTS AND DiscusSiONS}

\section{A. Inspection Improvement}

Fig. 5 demonstrated the SNR improvement by utilising the Golay and Barker sequences while the transducer is stationary and well-aligned on the sample surface. To characterise the performance of the coded excitations and validate their applications on the UAV, the recorded UAV single point inspections were repeated at various speeds, between $16 \%$ and $200 \%$ relative to the original recorded motion path (i.e. peak speeds of 20.51 to $256.42 \mathrm{~mm} / \mathrm{s}$ and 2.32 to $28.92 \%$ ). In all cases, the amplifier gain was set to $42 \mathrm{~dB}$ to maximise the received signal amplitude and prevent the signal becoming saturated in cases of ideal transducer alignment. The SNR improvements, and measurement errors acquired in each trial are plotted in Fig. 6 and Fig. 7 respectively.

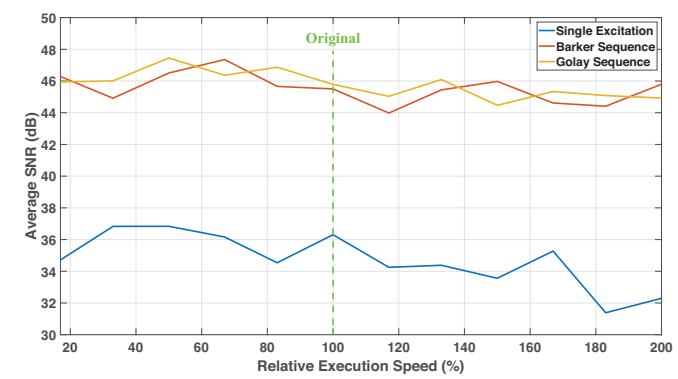

Fig. 6. SNR improvements across the inspection section while the transducer was stimulated by single pulse, Barker and Golay sequences under various motion speeds.

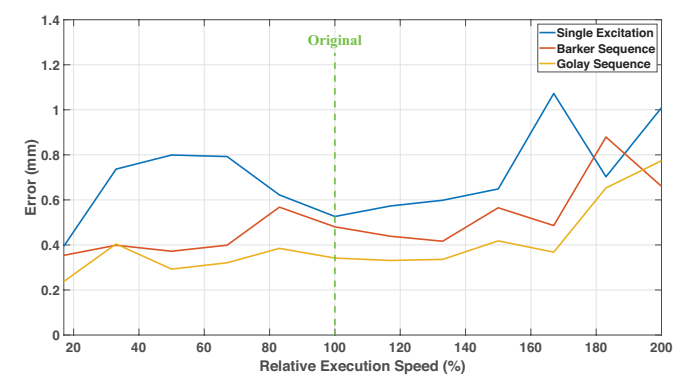

Fig. 7. Mean absolute errors across the inspection section while the transducer was stimulated by single pulse, Barker and Golay sequences under various motion speeds. 
As presented in Fig. 6, the SNR was significantly increased under the coded excitation schemas, leading to the improvements in the measurement accuracy shown in Fig. 7. The average measurement errors were reduced by a factor of two. Compared with those under the slower probe motions, the improvements at speeds higher than originally recorded from the UAV were not as significant.

Due to the fixed nature of the transceiver pulse repetition frequency when signal processing in real-time, slower speed trials acquired more readings. Where 254 thickness measurements were acquired at the lowest speed with single pulse excitation, only 19 measurements were captured under the highest speed motion path. Coded excitations require extra time for data processing so that the number or readings taken was reduced by $25 \%$ versus the single pulse excitation. In addition, more geometry features, including the boundaries of the thickness changes, can be captured when the probe moves slowly. A proportionately greater period of time is spent in regions where subsequent signals may be influenced by geometry that their earlier partner was not. The measurement errors at such boundaries were further increased owing to the observability of multiple reflections from differing backwalls in the A-scan signal.

\section{B. Signal Saturation}

During the UAV inspections, the gain of the amplifier in the ultrasonic transceiver circuit should be maximised to ensure the signals captured with large alignment errors are visible to the transceiver ADC (analogue to digital converter). As such, however, the high amplitude signals returned by a system with small alignment errors were overamplified to the point of saturation, resulting in significant distortion of the echo signals. These distortions lead to non-linear effects which cause harmonics of the base excitation frequency to appear, further impacting the waveform decoding.
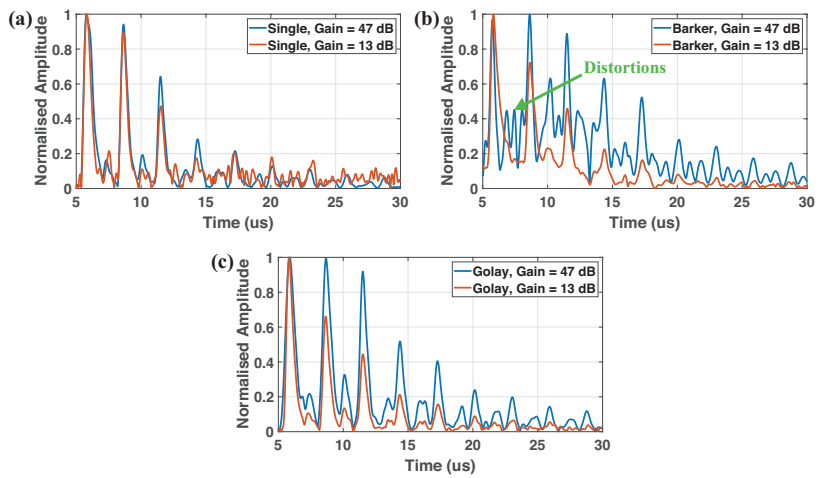

Fig. 8. A-scan signals while inspecting a $9 \mathrm{~mm}$ thickness sample. The transducer was excited by (a) single pulse excitation (b) 13-bit Barker sequence (c) 8-bit Golay sequence

As shown in Fig. 8, with the increase in the amplifier gain, the single pulse excitation and the Golay code presented a smaller distortion amplitude, and the echoes were still recognisable. However, the Barker code was overamplified at this maximum gain, leading to prominent distortions when compared to the single pulse and Golay excitation. Additionally, the second echo is hard to identify in the Barker code Ascan signal, owing to its masking by the presence of sidelobes and other phenomena appearing between the backwall echoes.

\section{Conclusions}

In an attempt to mitigate the effect of aerodynamic challenges on the quality of UAV based ultrasound inspection data, coded ultrasound excitations were proposed, and their implementation empirically evaluated. Under the coded excitations in the static case, $10.55 \mathrm{~dB}$ and $13.45 \mathrm{~dB}$ SNR improvements were observed in the Barker and Golay sequences respectively, leading to reduced measurement errors in scenarios where the transducer was in sub-optimal contact.

SNR improvements and reductions of the measurement errors were also observed in the non-static case when positioning the ultrasonic probe using the KUKA manipulator. The results demonstrated the capability of the coded excitation to reduce measurement errors when deployed aboard a UAV ultrasonic inspection system. The measurement errors were reduced by a factor of two when using the Golay coded excitations. This improvement was reduced at speeds above those recorded in the original UAV motion but consistently retained a positive effect on SNR relative to single pulse excitation throughout. Such results show that with adequate velocity control of the UAV flight path, Golay codes represent a valid strategy to improve the SNR when conducting contact-based ultrasonic measurements. Barker codes present a similar effect but of reduced improvement.

Finally, the impacts of the signal saturation on the coded excitation decoding process were described, highlighting the requirement for the autonomous adjustment of amplifier gain during the inspection, especially while using the Barker code.

\section{REFERENCES}

[1] R. A. Clark et al., "Autonomous and scalable control for remote inspection with multiple aerial vehicles," Rob. Auton. Syst., vol. 87, pp. 258-268, 2017.

[2] X. Li, Q. Yang, Z. Chen, X. Luo, and W. Yan, "Visible defects detection based on UAV-based inspection in large-scale photovoltaic systems," IET Renew. Power Gener., vol. 11, no. 10, pp. 1234-1244, 2017.

[3] R. Jarvis, F. Cegla, M. Kovac, and A. Farinha, "NDE sensor delivery using unmanned aerial vehicles," Insight Non-Destructive Test. Cond. Monit., vol. 60, no. 8, pp. 463-467, 2018.

[4] D. Zhang, R. Watson, G. Dobie, C. MacLeod, and G. Pierce, "Autonomous Ultrasonic Inspection Using Unmanned Aerial Vehicle," IEEE Int. Ultrason. Symp. IUS, vol. 2018-October, 2018.

[5] I. A. Veres et al., "Golay code modulation in low-power laserultrasound," Ultrasonics, vol. 53, no. 1, pp. 122-129, 2013.

[6] R. Su et al., "Coded excitation for air-coupled ultrasound phased arrays," 56th Annu. Conf. Br. Inst. Non-Destructive Testing, NDT 2017, 2017.

[7] B. Zhou, D. M. Green, and J. C. Middlebrooks, "Characterization of external ear impulse responses using Golay codes.," J. Acoust. Soc. Am., vol. 92, no. 2, pp. 1169-71, 1992.

[8] M. M. Daffalla and A. A. Babiker, "Adaptive Coding, Modulation and Filtering of Radar Signals," in Topics in Radar Signal Processing, InTech, 2018.

[9] GB Inspection Systems Ltd., "Compression Wave $0^{\circ}$ Probes," 2019. [Online]. Available: https://www.gbinspection.com/products/probesaccessories/compression-wave-0-probes. [Accessed: 20-Sep-2019].

[10] C. Mineo et al., "Enabling robotic adaptive behaviour capabilities for new industry 4.0 automated quality inspection paradigms,' NDT 2018 - 57th Annu. Conf. Br. Inst. Non-Destructive Test., pp. 28-39, 2018. 